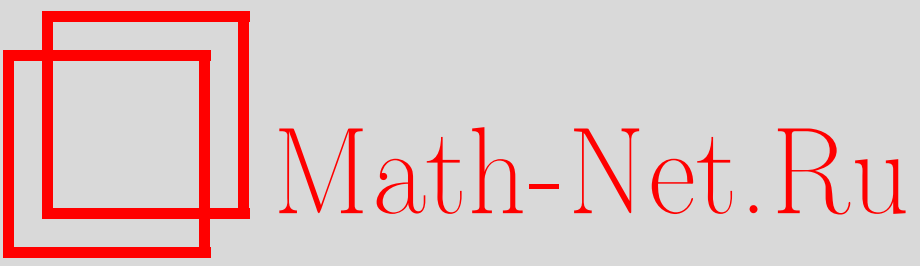

Ю. В. Чугреев, О принципе причинности для рейсснер-нордстремовского поля в РТГ. Случай малых зарядов $q^{2} \leq m^{2}, T M \Phi, 2002$, том 132, номер 3, 475-483

DOI: https://doi.org/10.4213/tmf375

Использование Общероссийского математического портала Math-Net.Ru подразумевает, что вы прочитали и согласны с пользовательским соглашением

http://www. mathnet.ru/rus/agreement

Параметры загрузки:

IP : 54.162 .127 .20

26 апреля 2023 г., 16:54:31 


\section{О ПРИНЦИПЕ ПРИЧИННОСТИ ДЛЯ РЕЙССНЕР-НОРДСТРЕМОВСКОГО ПОЛЯ В РТГ. СЛУЧАЙ МАЛЫХ ЗАРЯДОВ $q^{2} \leqslant m^{2}$}

Проведен анализ соблюдения принципа причинности для внешнего электровакуумного решения, создаваемого статическим сферически-симметричным электрически заряженным телом в релятивистской теории гравитации с нулевой массой гравитона (рейсснер-нордстремовское решение). Показано, что этот принцип ограничивает значения константы, содержашейся во внешнем решении, а также устанавливает нижний предел для радиуса источника. Установлено, что внешнее поле сверхплотных звездных конфигураций удовлетворяет принципу причинности.

Ключевые слова: релятивистская теория гравитации, принцип причинности, решение Рейсснера-Нордстрема.

\section{1. ВВЕДЕНИЕ}

Рейсснер-нордстремовское решение в релятивистской теории гравитации (РТГ) (с нулевой массой гравитона) описывает точное внешнее электровакуумное поле статического сферически-симметричного электрически заряженного источника. Это решение впервые было получено Выблым [1] и позднее независимо Шошем (E. Soos) и Ионеску (D. Ionescu):

$$
\begin{aligned}
& d s^{2}=g_{\mu \nu} d x^{\mu} d x^{\nu}=\left(1-\frac{2 m}{r}+\frac{q^{2}}{r^{2}}\right) d t^{2}-\frac{d r^{2}}{1-\frac{2 m}{r}+\frac{q^{2}}{r^{2}}}-r^{2} d \Omega^{2} \\
& d \sigma^{2}=\gamma_{\mu \nu} d x^{\mu} d x^{\nu}=d t^{2}-R^{\prime 2} d r^{2}-R^{2} d \Omega^{2}
\end{aligned}
$$

где

$$
\begin{array}{ll}
R(r)=r-m+C_{1}\left(1+\frac{r-m}{2 p} \ln \left|\frac{r-m-p}{r-m+p}\right|\right), & m^{2}>q^{2}, \\
R(r)=r-m-\frac{C_{2}}{(r-m)^{2}}, & m^{2}=q^{2} .
\end{array}
$$

\footnotetext{
* Московский государственный университет, Москва, Россия
} 
Здесь

$$
R^{\prime} \equiv \frac{d R(r)}{d r}, \quad d \Omega^{2} \equiv d \theta^{2}+\sin ^{2}(\theta) d \varphi^{2}, \quad p \equiv \sqrt{m^{2}-q^{2}},
$$

$\gamma_{\mu \nu}$ - метрический тензор пространства Минковского, $g_{\mu \nu}$ - метрика эффективного риманова пространства, $R$ - радиальная координата в пространстве Минковского, $r$ шварцшильдова радиальная переменная, $C_{1}, C_{2}$ - произвольные постоянные. Отметим также, что простейший случай рейсснер-нордстремовского решения, соответствуюший выбору $C_{1}=0\left(m^{2}>q^{2}\right)$, рассматривался в статье [2], а в работе [3] было показано, что это решение удовлетворяет принципу причинности. Целью настояшей работы является проведение анализа соблюдения принципа причинности для рейсснер-нордстремовского решения РТГ обшего вида (1), (2). Случай больших зарядов $m^{2}<q^{2}$ более сложен. Он будет рассмотрен отдельно.

Впервые принцип причинности для сферически-симметричного решения РТГ был рассмотрен в работе [4] при $q^{2}=0$. Примененная методика включала в себя поиск ограничений на константу $C_{1}$, при которых все возможные геодезические линии риманова пространства удовлетворяли принципу причинности во всей области допустимых значений, определяемой константой $C_{1}$. Этот метод был довольно громоздким, так как варьированию подвергались параметры геодезических линий. В данной работе применяется более простая процедура проверки принципа причинности, впервые использованная для керр-ньюменовского решения РТГ [5]. А именно, варьируются не параметры геодезических линий, а компоненты самого изотропного вектора, входящего в определение принципа причинности. При этом не возникает необходимости решать уравнения геодезических.

Выведем соответствуюшие условия в обшем сферически-симметричном случае. Согласно принципу причинности любой изотропный в римановом пространстве-времени вектор $u^{\mu}$ должен быть времениподобным в пространстве Минковского:

$$
\begin{aligned}
& g_{\mu \nu} u^{\mu} u^{\nu}=0, \\
& \gamma_{\mu \nu} u^{\mu} u^{\nu}>0 .
\end{aligned}
$$

Вводя компоненты $u^{\mu}=\left\{u^{t}, u^{r}, u^{\theta}, u^{\varphi}\right\}$, из уравнения (4) получаем

$$
g_{t t}\left(u^{t}\right)^{2}+g_{r r}\left(u^{r}\right)^{2}+g_{\theta \theta}\left[\left(u^{\theta}\right)^{2}+\sin ^{2}(\theta)\left(u^{\varphi}\right)^{2}\right]=0 .
$$

Отсюда следует, что

$$
\left(\frac{u^{r}}{u^{t}}\right)^{2}+\sin ^{2} \theta\left(\frac{u^{\varphi}}{u^{t}}\right)^{2}=-\frac{g_{t t}}{g_{\theta \theta}}-\frac{g_{r r}}{g_{\theta \theta}}\left(\frac{u^{r}}{u^{t}}\right)^{2} .
$$

Подставляя левую часть в неравенство (5) и обозначая $u^{r} / u^{t} \equiv e^{r}$, находим

$$
\gamma_{t t}-g_{t t} \frac{\gamma_{\theta \theta}}{g_{\theta \theta}}+\left(e^{r}\right)^{2}\left(\gamma_{r r}-g_{r r} \frac{\gamma_{\theta \theta}}{g_{\theta \theta}}\right) \geqslant 0 .
$$


Это неравенство должно выполняться при любом допустимом значении $e^{r}$. Поскольку функция зависит от $\left(e^{r}\right)^{2}$ монотонно, то согласно принципу максимума она достигает экстремумов на границах интервала допустимых значений $\left(e^{r}\right)^{2}$, который можно получить из уравнения (6):

$$
0 \leqslant\left(e^{r}\right)^{2} \leqslant-\frac{g_{t t}}{g_{r r}} .
$$

Таким образом, мы получаем два условия общего вида, определяющих соблюдение принципа причинности для сферически-симметричной задачи:

$$
\begin{aligned}
& g_{\theta \theta} \gamma_{t t} \leqslant g_{t t} \gamma_{\theta \theta}, \\
& g_{r r} \gamma_{t t} \leqslant g_{t t} \gamma_{r r} .
\end{aligned}
$$

Неравенства (9), (10) совпадают с полученными в монографиии [6] условиями для простейшего выбора координат $\gamma_{t t}=-\gamma_{r r}=1, \gamma_{\theta \theta}=r^{2}$. В дальнейшем мы будем для проверки принципа причинности рассматривать соблюдение именно условий (9), (10).

\section{2. ПРИНЦИП ПРИЧИННОСТИ ДЛЯ СЛУЧАЯ $q^{2} \leqslant m^{2}$}

Рассмотрим вначале общий характер и область допустимых значений для случая (1). График зависимости радиуса пространства Минковского $R$ от шваршшильдовой радиальной переменной $r$ представлен на рис. 1 .

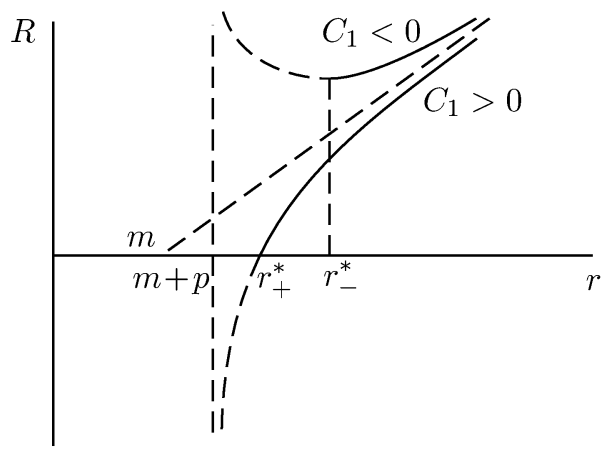

Рис. 1

Все кривые с отрицательными $C_{1}$ имеют минимум в точке $r_{-}^{*}$, лежашей выше сферы горизонта событий с радиусом $(m+p): r_{-}^{*}>m+p$. Очевидно, что область допустимых значений отвечает лучу $r>r_{-}^{*}$. Аналогично в случае положительных $C_{1}$ переменная $r$ также не может достигнуть значения $(m+p): r>r_{+}^{*}$, где в точке $r_{+}^{*}>(m+p)$ обрашается в нуль радиус пространства Минковского: $R\left(r_{+}^{*}\right)=0$. Такая же картина имеет место и для случая $m^{2}=q^{2}$ : отрицательным значениям $C_{2}$ отвечают кривые с локальным минимумом, лежашим выше радиуса сингулярности преобразования, а положительным кривые, оканчиваюшиеся на горизонтальной оси $R=0$. 
2.1. Рассмотрим случай отрицательных значений $C_{1}$. Условие $(10)$ в этом случае представим в виде

$$
\left(1-\frac{2 m}{r}+\frac{q^{2}}{r^{2}}\right)^{-2} \geqslant R^{\prime 2} .
$$

Очевидно, что оно выполняется во всей области допустимых значений, так как при $m^{2}>$ $q^{2}$ справедливы неравенства

$$
\left(1-\frac{2 m}{r}+\frac{q^{2}}{r^{2}}\right)^{-1} \geqslant \frac{r^{2}}{(r-m)^{2}}>1,
$$

а $R^{\prime}<1$, что видно из рис. 1 .

Проверим теперь условие (9), представив его в виде

$$
r^{2} \geqslant\left(1-\frac{2 m}{r}+\frac{q^{2}}{r^{2}}\right) R^{2}(r)
$$

Поскольку асимптотика $R(r)$ при больших $r$ имеет вид

$$
R(r)=r-m-\frac{1}{3} C_{1} \frac{p^{2}}{r^{2}}+O\left(\frac{1}{r^{3}}\right),
$$

то в этой области неравенство (12) выполняется:

$$
r^{2}>r^{2}\left(1-\frac{4 m}{r}+O\left(\frac{1}{r^{2}}\right)\right) .
$$

В силу монотонности $R(r)$ при $r>r_{-}^{*}$ для того, чтобы неравенство (12) было справедливым, достаточно добиться этого на границе, в точке $r=r_{-}^{*}$ :

$$
R^{\prime}\left(r_{-}^{*}\right)=0
$$

Так как

$$
R^{\prime}(r)=1+C_{1}\left(\frac{1}{2 p} \ln \frac{r-m-p}{r-m+p}+\frac{r-m}{(r-m)^{2}-p^{2}}\right),
$$

то из условий (12), (13) находим

$$
\left|C_{1}\right| \leqslant\left(\frac{r_{-}^{*}}{p}\right)^{2} \sqrt{\left(r_{-}^{*}-m\right)^{2}-p^{2}} .
$$

Условие (13) вместе с неравенством (15) позволяют установить интервал изменения отрицательной константы $C_{1}$, обеспечивающий соблюдение принципа причинности решения во всей области допустимых значений $r \geqslant r_{-}^{*}$. Обозначим этот интервал для фиксированного значения заряда $q$ как $C_{1}^{-} \leqslant C_{1}<0$. Значение $r_{-}^{*}$, отвечаюшее предельному значению $C_{1}^{-}$, можно найти из уравнения

$$
\left[\frac{1}{2 p} \ln \left(\frac{r_{-}^{*}-m-p}{r_{-}^{*}-m+p}\right)+\frac{r_{-}^{*}-m}{\left(r_{-}^{*}-m\right)^{2}-p^{2}}\right] \frac{\left(r_{-}^{*}\right)^{2}}{p^{2}} \sqrt{\left(r_{-}^{*}-m\right)^{2}-p^{2}}=1 .
$$


В частном случае $q=0$ мы получим $p=m$, а уравнение (16) можно представить в виде

$$
\left[\frac{1}{2} \ln \frac{x-2}{x}+\frac{x-1}{x(x-2)}\right] x^{2} \sqrt{x^{2}-2 x}=1 .
$$

Здесь $x \equiv r_{-}^{*} / m$. Это уравнение, как и следовало ожидать, совпадает с уравнением для $r_{-}^{*}$, полученным в работе [4] по другой методике. Его решение легко найти численно:

$$
\frac{C_{1}^{-}}{m}=-162.202 \ldots, \quad \frac{r_{-}^{*}}{m}=5.8477 \ldots
$$

Решения со значениями $C_{1}$ в интервале $C_{1}^{-} \leqslant C_{1} \leqslant 0$ удовлетворяют принципу причинности для случая $q=0$.

Уравнение (16) трансцендентное. Для дальнейшего анализа его удобно записать в безразмерных величинах $x \equiv r_{-}^{*} / m, \rho \equiv p / m, 0 \leqslant \rho \leqslant 1$ :

$$
\left[\frac{1}{2 \rho} \ln \frac{x-1-\rho}{x-1+\rho}+\frac{x-1}{(x-1)^{2}-\rho^{2}}\right] \frac{x^{2}}{\rho^{2}} \sqrt{(x-1)^{2}-\rho^{2}}=1 .
$$

Решения этого уравнения представлены графически на рис. 2 и 3.

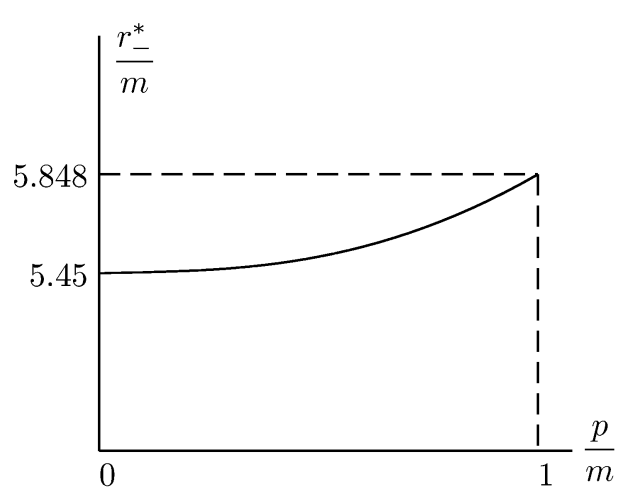

Рис. 2

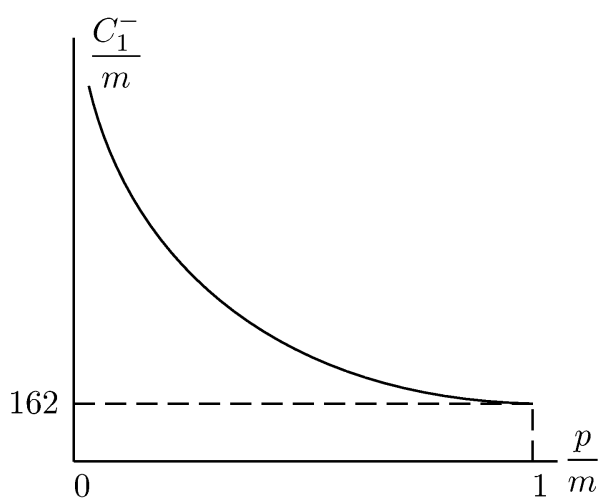

Рис. 3

Зависимость $x=x(\rho)$, как следует из рис. 2 , очень слабая, в отличие от $C_{1}^{-}=C_{1}^{-}(\rho)$, представленной на рис. 3. При $\rho \rightarrow 0\left(q^{2} \rightarrow m^{2}\right)$ значение $\left|C_{1}^{-}\right|$быстро растет, а его асимптотика в этой области имеет вид

$$
\frac{C_{1}^{-}}{m} \rightarrow-\frac{3}{2} \frac{(x-1)^{3}}{\rho^{2}}
$$

Таким образом, электровакуумная область причинности для любого заряда тела, не превышаюшего его массы $\left(q^{2}<m^{2}\right)$, в случае отрицательной константы $C_{1}$ не может иметь радиус меньше некоторого предельного, равного $\approx 5.4495 \mathrm{~m}$, что соответствует случаю $q=m$ (см. ниже раздел 3 ). Результат довольно неожиданньй, если принять во 
внимание то, что этот предельно малый радиус более чем в два раза превосходит радиус рейсснер-нордстремовского горизонта $m+\sqrt{m^{2}-q^{2}} \leqslant 2 m$. Сформулируем этот вывод в несколько ином виде: если внутреннее решение задачи со статическим электрически заряженным источником сшивается с внешним полем, содержашим отрицательную константу $C_{1}$, то радиус этого тела должен превьшать $\approx 5.4495 \mathrm{~m}$. В случае $C_{1}>0$ мы получим аналогичный вывод, однако величина предельного минимального радиуса тела будет меньше.

2.2. Перейдем к подробному рассмотрению случая положительных значений константы $C_{1}$. Вновь необходимо проанализировать соблюдение условий $(9),(10)$, вытекаюших из приншипа причинности. Очевидно, что условие (9), записанное в форме неравенства (12)

$$
r^{2} \geqslant\left(1-\frac{2 m}{r}+\frac{q^{2}}{r^{2}}\right) R^{2}(r) \geqslant\left(1-\frac{m}{r}\right)^{2} R^{2}(r),
$$

справедливо во всей области допустимых значений $\infty \geqslant r \geqslant r_{+}^{*}$, поскольку $R(r)<r-m$. Проверим теперь условие (10), записанное в виде неравенства (11).

Поскольку асимптотика $R^{\prime}$ при больших $r$ имеет вид

$$
R^{\prime}(r)=1+\frac{2}{3} C_{1} \frac{p^{2}}{r^{3}}+O\left(\frac{1}{r^{4}}\right)
$$

то условие (11) в этой области выполняется, так как

$$
\left(1-\frac{2 m}{r}+\frac{q^{2}}{r^{2}}\right)^{-1}=1+\frac{2 m}{r}+O\left(\frac{1}{r^{2}}\right) .
$$

В силу монотонности $R^{\prime}$ в области $\infty \geqslant r \geqslant r_{+}^{*}$ для того, чтобы неравенство (11) было справедливым, достаточно добиться этого на границе, в точке $r=r_{+}^{*}$ такой, что

$$
R\left(r_{+}^{*}\right)=0
$$

Отсюда и из неравенства (11) находим

$$
C_{1} \leqslant\left(\frac{r_{+}^{*}}{p}\right)^{2} \frac{\left(r_{+}^{*}-m\right)^{2}}{p^{2}}
$$

Уравнение (19) вместе с неравенством (11) позволяют установить интервал изменения положительной константы $C_{1}$, обеспечивающий соблюдение принципа причинности во всей области допустимых значений. Обозначим этот интервал для фиксированного значения заряда как $0<C_{1} \leqslant C_{1}^{+}$. Значение $r_{+}^{*}$, отвечаюшее предельному значению $C_{1}^{+}>0$, можно найти из уравнения

$$
\left[\frac{r_{+}^{*}-m}{2 p} \ln \left(\frac{r_{+}^{*}-m-p}{r_{+}^{*}-m+p}\right)+1\right] \frac{\left(r_{+}^{*}\right)^{2}}{p^{2}}=-1 .
$$


В частном случае нулевого заряда $(p=m)$ это уравнение можно представить в виде

$$
\left[\frac{x-1}{2} \ln \frac{x-2}{x}+1\right] x^{2}+1=0,
$$

где $x \equiv r_{+}^{*} / m$. Это уравнение, как и в случае отрицательных $C_{1}$, совпадает с уравнением для $r_{+}^{*}$, полученным в работе [4] по другой методике. Его решение легко найти численно:

$$
\frac{C_{1}^{+}}{m}=14.0986 \ldots, \quad \frac{r_{+}^{*}}{m}=2.7981 \ldots
$$

Решения со значениями $C_{1}$ в интервале $0 \leqslant C_{1} \leqslant C_{1}^{+}$и $r$ в области $\infty \geqslant r \geqslant r_{+}^{*}$, где $r_{+}^{*}$ отвечает соответствующему $C_{1}^{+}$, удовлетворяют принципу причинности для случая $q=0$. Обший случай определяется уравнением $(21)$, которое удобно переписать с использованием безразмерных величин $x \equiv r_{+}^{*} / m, \rho \equiv p / m, 0 \leqslant \rho \leqslant 1$ :

$$
\left[\frac{x-1}{2 \rho} \ln \frac{x-1-\rho}{x-1+\rho}+1\right] \frac{x^{2}}{\rho^{2}}=-1 \text {. }
$$

Решения этого трансцендентного уравнения представлены графически на рис. 4.

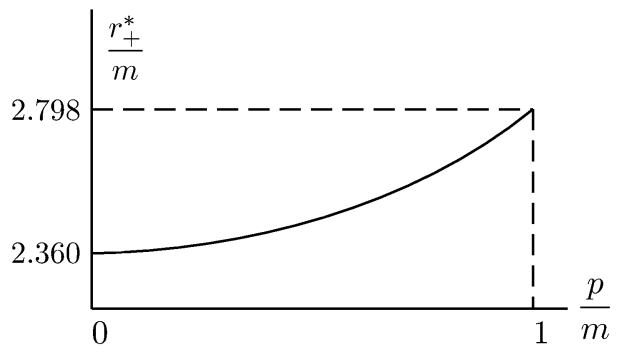

Рис. 4

Так же как и для случая отрицательных $C_{1}$, зависимость $x=x(\rho)$ очень слабая и существует минимальное значение $x=2.360$. Напротив, соответствующие значения (положительные) $C_{1}^{+}$изменяются сильно: от бесконечности при $\rho=0$ до 14.1 при $\rho=1$, как показано на рис. 5 .

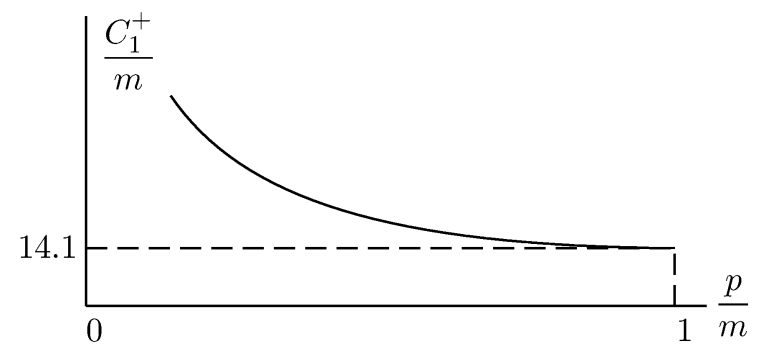

Рис. 5 
При $\rho \rightarrow 0\left(q^{2} \rightarrow m^{2}\right)$ значение $C_{1}^{+} / m$ быстро растет и имеет асимптотику

$$
\frac{C_{1}^{+}}{m} \rightarrow \frac{3(x-1)^{3}}{\rho^{2}}
$$

Аналогично случаю отрицательных $C_{1}$ можно сделать вывод, что при положительном значении $C_{1}$ тело не может иметь радиус меньше $2.360 \mathrm{~m}$, что превышает радиус рейсснер-нордстремовского горизонта.

\section{3. ПРИНЦИП ПРИЧИННОСТИ ДЛЯ СЛУЧАЯ $q^{2}=m^{2}$}

Этот случай рассматривается аналогично предыдущему случаю малых зарядов $\left(q^{2}<m^{2}\right)$. Анализ упрощается, так как все уравнения легко решаются в явном виде. В результате принципу причинности удовлетворяют решения во всей области допустимых значений $r$, если только константа $C_{2}$ находится внутри интервала

$$
-44.0454 \ldots=-\frac{\sqrt{2 / 3}}{3(1-\sqrt{2 / 3})^{3}} \leqslant \frac{C_{2}}{m^{3}} \leqslant \frac{1}{8} \text {. }
$$

Для $C_{2} / m^{3}=1 / 8$ соответствуюшее предельное значение $r_{+}^{*}$ равно $3 m / 2$, а для наименьшего отрицательного значения $C_{2}$ можно найти, что $r_{-}^{*} / m=1 /(1-\sqrt{2 / 3}) \approx$ 5.4495 .

Отметим, что как в случае $q^{2}<m^{2}$, так и в случае $q^{2}=m^{2}$ область допустимых отрицательных значений констант $C_{1}$ и $C_{2}$ значительно больше области их положительных значений, тогда как область допустимых значений $r_{-}^{*}$ во всех случаях заметно превышает область $r_{+}^{*}$.

Рассмотрим теперь оставшийся случай $C_{2}=0$. Область допустимых значений этого решения

$$
\begin{aligned}
d s^{2} & =\frac{(r-m)^{2}}{r^{2}} d t^{2}-\frac{r^{2} d r^{2}}{(r-m)^{2}}-r^{2} d \Omega^{2}, \\
d \sigma^{2} & =d t^{2}-d r^{2}-(r-m)^{2} d \Omega^{2}
\end{aligned}
$$

есть $m \leqslant r<\infty$. Отметим, что в этом случае имеется полюс второго порядка для $g_{r r}$ и нуль второго порядка для $g_{t t}$ на рейсснер-нордстремовском горизонте $r=m$. Эта сқера в шварцшильдовых координатах отвечает точке начала отсчета в сферических координатах пространства Минковского.

Условия причинности (9), (10) представимы в виде

$$
r^{2} \geqslant \frac{(r-m)^{4}}{r^{2}}, \quad \frac{r^{2}}{(r-m)^{2}} \geqslant 1 .
$$

Они выполняются во всей области допустимых значений, следовательно, решение (24) удовлетворяет принципу причинности. 
О ПРИНЦИПЕ ПРИЧИННОСТИ ДЛЯ РЕЙССНЕР-НОР ДСТРЕМОВСКОГО ПОЛЯ В РТГ 483

\section{4. ПРОВЕРКА СОБЛЮДЕНИЯ ПРИНЦИПА ПРИЧИННОСТИ ДЛЯ ВНЕШНЕГО ПОЛЯ СВЕРХПЛОТНЫХ ЗВЕЗД}

В работе [7] Авакяном была приведена таблица параметров сверхплотных звездных конфигураций, состоящих из реального газа барионов. Эти значения были получены в результате численного интегрирования внутреннего решения РТГ и последуюшего сшивания с вакуумным решением (1) для случая $q=0$ и $C_{1} \neq 0$. Покажем, что для таких конфигураций вся вакуумная область удовлетворяет принципу причинности. Представим данные работы [7] в виде табл. 1 , где через $r_{S}$ обозначен радиус звезды в шварцильдовых координатах, константа $C_{1}$ положительна.

ТАБЛИЦА 1

\begin{tabular}{|c|c|c|c|c|}
\hline$\rho_{c}\left(г / \mathrm{cM}^{3}\right)$ & $3.55 \cdot 10^{15}$ & $6.81 \cdot 10^{15}$ & $9.79 \cdot 10^{15}$ & $1.69 \cdot 10^{16}$ \\
\hline$m / m_{\odot}$ & 0.35 & 0.77 & 1.05 & 1.39 \\
\hline$r_{S}(\mathrm{KM})$ & 16.16 & 12.86 & 12.06 & 10.49 \\
\hline$C_{1}(\mathrm{KM})$ & 2.9 & 2.8 & 2.6 & 2.2 \\
\hline
\end{tabular}

Чтобы доказать причинность вакуумной области этих решений, достаточно убедиться, что для всех этих конфигураций $C_{1} / m<14.1$, а радиус звезды $r_{S}$ превышает значение $r_{+}^{*}$, отвечающее данному выбору $C_{1}$. Объединив эти величины в табл. 2, мы приходим к заключению, что представленные в работе [7] решения удовлетворяют принципу причинности во внешней области.

ТАБЛИЦА 2

\begin{tabular}{|c|c|c|c|c|}
\hline$C_{1} / m$ & 11 & 4.6 & 3.5 & 2.2 \\
\hline$r_{S}(\mathrm{KM})$ & 16.16 & 12.86 & 12.06 & 10.49 \\
\hline$r_{+}^{*}(\mathrm{KM})$ & 0.7 & 1.4 & 1.7 & 2.2 \\
\hline
\end{tabular}

Благодарности. Автор выражает благодарность А. А. Логунову, М. А. Мествиришвили и Д. Ионеску за полезные обсуждения.

\section{Список литературы}

[1] Ю. П. Выбльй. Поле точечного электрического заряда в релятивистской теории гравитации. В сб.: Точные решения уравнений Эйнштейна и их физическая интерпретация. Ред. И. Р. Пийр. Тарту: Изд-во Тартусского ун-та, 1988. С. 74-76.

[2] П. В. Карабут, Ю. В. Чугреев. ТМФ. 1989. Т. 78. № 2. С. 305-313.

[3] П. В. Карабут, Ю. В. Чугреев. ТМФ. 1990. Т. 83. № 3. С. 470-475.

[4] П. В. Карабут, Ю. В. Чугреев. ТМФ. 1990. Т. 84. № 3. С. 474-480.

[5] Ю. В. Чугреев. ТМФ. 1991. Т. 88. № 3. С. 459-466.

[6] А. А. Логунов. Теория гравитационного поля. М.: Наука, 2000.

[7] Р. М. Авакян. О решении для изолированной массы в гармонических координатах. В сб.: Точные решения уравнений Эйнштейна и их физическая интерпретация. Ред. И. Р. Пийр. Тарту: Изд-во Тартусского ун-та, 1988. С. 22-24. 